\title{
Weighted Multiple Model Adaptive Control for a Category of Systems with Colored Noise
}

\author{
Yuzhen Zhang \\ School of Automation and Electrical Engineering, University of Science and Technology Beijing, 30 Xueyuan Road \\ Haidian District, Beijing, China \\ Weicun Zhang \\ School of Automation and Electrical Engineering, University of Science and Technology Beijing, 30 Xueyuan Road \\ Haidian District, Beijing, China \\ Qing Li \\ School of Automation and Electrical Engineering, University of Science and Technology Beijing, 30 Xueyuan Road \\ Haidian District, Beijing, China \\ E-mail: zhangyz_ustb@163.com,weicunzhang@ustb.edu.cn,liqing@ies.ustb.edu.cn \\ www.ustb.edu.cn
}

\begin{abstract}
The multiple model adaptive control (MMAC) of discrete-time stochastic system with colored noise is considered in this paper. Model set contains one adaptive identification and some fixed models. Based on the output errors of the models, a simple weighting algorithm is adopted with guaranteed convergence. The proofs of the stability and convergence of system are presented. Besides, the influence of initial value of adaptive model on system performance is described. Finally, computer simulation results can verify the theoretical results.
\end{abstract}

Keywords: discrete-time; colored noise; multiple model adaptive control; stability; convergence.

\section{Introduction}

As the largely change of system parameters makes the dynamical system become complicated. The classical adaptive control can't meet the requirements. Therefore, MMAC developed. The most suitable controller is used to control the system ${ }^{1}$.

The weighted multiple model adaptive control (WMMAC) was researched in 1960's ${ }^{2}$. In that system, Kalman filter and posterior possibility evaluator (PPE) algorithm are important ${ }^{3}$. Later, K. S. Narendra put forward the concept of switching multiple model adaptive control ${ }^{4,5}$. To some extent, WMMAC can be regarded as a kind of slow switching ${ }^{4,5}$. Therefore, the scheme can alleviate the vibration when switching.

However, the system stability analysis of the WMMAC has been plaguing the researchers all the time. In Refs. 4 and 6, the stability and convergence are proved based on the virtual equivalent system (VES) theory. But, its model set just includes fixed models. If the real model isn't in the model set, the system is not good.

Considering the above problems, in this paper, a finite number of fixed and one adaptive identification models are designed to cover the discrete-time stochastic system with colored noise.

\section{Description of WMMAC}

For analyzing system clearly, a block diagram of a general discrete-time WMMAC system is shown in Fig.1.

The meanings of symbols are shown: $P$ is the uncertainty plant; $C_{i}$ is a local controller corresponded 
to the fixed model; $C_{\theta}$ is the local controller corresponded to the adaptive identification model; $p_{i}(k)$ is weighted value for its local controller; $p_{\theta}(k)$ is the weighted value for the adaptive identification controller. Through the algorithm, the weighted values are known. Then, the $u(k)$ can be obtained by weighted sum.

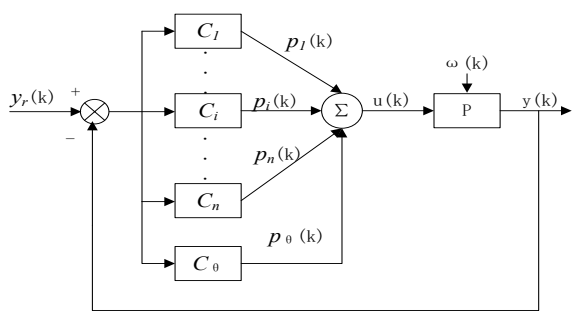

Fig. 1. Concise block diagram of a general WMMAC system

Consider the following discrete-time dynamical plant, the mathematical model is

$$
\begin{aligned}
A\left(z^{-1}\right) y(k) & =z^{-d} B\left(z^{-1}\right) u(k)+C\left(z^{-1}\right) \omega(k) \\
& =z^{-d} B\left(z^{-1}\right) u(k)+v(k)
\end{aligned}
$$

where

$$
\left\{\begin{array}{l}
A\left(z^{-1}\right)=1+a_{1} z^{-1}+a_{2} z^{-2}+\cdots+a_{n_{a}} z^{-n_{a}} \\
B\left(z^{-1}\right)=b_{0}+b_{1} z^{-1}+b_{2} z^{-2}+\cdots+b_{n_{b}} z^{-n_{b}} \\
C\left(z^{-1}\right)=1+c_{1} z^{-1}+c_{2} z^{-2}+\cdots+c_{n_{c}} z^{-n_{c}} \\
v=C\left(z^{-1}\right) \omega(k)
\end{array}\right.
$$

$y(k)$ and $u(k)$ are the output and the input. The $\omega(k)$ is a zero-mean white noise. The $v(k)$ is colored noise.

The plant also can be written as

$$
y(k)=\varphi^{T}(k-d) \theta+\omega(k)
$$

where

$$
\begin{gathered}
\varphi(k-d)=\left[-y(k-1), \cdots,-y\left(k-n_{a}\right), u(k-d), \cdots, u\left(k-d-n_{b}\right),\right. \\
\left.\omega(k-1), \cdots, \omega\left(k-n_{c}\right)\right]^{\mathrm{T}} \in R^{\left(n_{a}+n_{b}+1+n_{c}\right) \times 1} \\
\theta=\left[a_{1}, \cdots, a_{n_{a}}, b_{0}, \cdots, b_{n_{b}}, c_{1}, \cdots, c_{n_{c}}\right]^{\mathrm{T}} \in R^{\left(n_{a}+n_{b}+1+n_{c}\right) \times 1}
\end{gathered}
$$

$\mathrm{M}=\left\{M_{i}, M_{\theta}, i=1,2, \cdots, N\right\}$ is the model set. For every fixed model, the corresponded controller is designed by pole placement control. At the same time, the one adaptive identification model is designed by indirect pole assignment self-tuning control. The recursive extended least square is used to estimated parameter ${ }^{7}$. That is

$$
\left\{\begin{array}{l}
\hat{\theta}(k)=\hat{\theta}(k-1)+K(k)\left[y(k)-\hat{\varphi}^{\mathrm{T}}(k) \hat{\theta}(k-1)\right] \\
K(k)=\frac{P(k-1) \hat{\varphi}(k)}{1+\hat{\varphi}^{\mathrm{T}}(k) P(k-1) \hat{\varphi}(k)} \\
P(k)=\left[\mathrm{I}-K(k) \hat{\varphi}^{\mathrm{T}}(k)\right] P(k-1)
\end{array}\right.
$$

where

$$
\left\{\begin{aligned}
\hat{\varphi}(k)= & {\left[-y(k-1), \cdots,-y\left(k-n_{a}\right), u(k-d), \cdots, u\left(k-d-n_{b}\right)\right.} \\
& \left., \hat{\omega}(k-1), \cdots, \hat{\omega}\left(k-n_{c}\right)\right]^{\mathrm{T}} \\
\hat{\theta}=\left[\hat{a}_{1}, \cdots, \hat{a}_{n_{a}}, \hat{b}_{0}, \cdots, \hat{b}_{n_{b}}, \hat{c}_{1}, \cdots, \hat{c}_{n_{c}}\right]^{\mathrm{T}} & \\
\hat{\omega}(k)= & y(k)-\hat{y}(k)=y(k)-\hat{\varphi}(k)^{\mathrm{T}} \hat{\theta}
\end{aligned}\right.
$$

As initial values will affect the system performance, we will discuss that specifically in simulation studies.

For each local model, its output $y_{i}(k)$ is used to define the output error $e_{i}(k)$. As shown in Fig.1, the global control $u(k)$ is obtained by

$$
u(k)=\sum_{i=1}^{N+1} p_{i}(k) u_{i}(k)
$$

In this paper, the algorithm ${ }^{4}$ to calculate weight is

$$
\begin{gathered}
p_{i}(0)=l_{i}(0)=\frac{1}{N+1} \\
l_{i}^{\prime}(k)=\alpha+\frac{1}{k} \sum_{j=1}^{k} e_{i}^{2}(j) ; e_{i}(k)=y(k)-y_{i}(k)
\end{gathered}
$$

where $\alpha$ is 0.001 , avoiding $l_{i}^{\prime}(k)=0$.

$$
\begin{gathered}
l_{\min }^{\prime}(k)=\min _{i}\left\{l_{i}^{\prime}(k)\right\} \\
l_{i}(k)=\frac{l_{\min }^{\prime}(k)}{l_{i}^{\prime}(k)} l_{i}(k-1) \\
p_{i}(k)=\frac{l_{i}(k)}{\sum_{j=1}^{N+1} l_{j}(k)}
\end{gathered}
$$

The convergence character is presented in Ref. 4 .

In the computer simulation, in order to prevent the weight converge to zero, we can limit the threshold value.

\section{Stability and Convergence of the System}

On the basis of $\mathrm{VES}^{4,6}$, this section gives the stability proof of the WMMAC system.

\subsection{WMMAC for LTI plant}

Theorem 1. If a WMMAC system satisfies: 
- $\quad$ The true model of plant, say $M_{j}$, is included in the fixed models of the model set $\mathrm{M}$;

- Model $M_{j}$ generates with probability one the minimum output error in the sense that

$$
\left\{\begin{array}{c}
\sum_{r=1}^{k} e_{j}^{2}(r)<\sum_{r=1}^{k} e_{i}^{2}(r), \forall k \geq d+1, i \neq j \\
\frac{1}{k} \sum_{r=1}^{k} e_{j}^{2}(r) \rightarrow R_{j} ; \frac{1}{k} \sum_{r=1}^{k} e_{i}^{2}(r) \rightarrow R_{i}
\end{array}\right.
$$

where $R_{j}$ is a constant, $R_{i}$ may be constant or infinity, $R_{j}<R_{i}, i \neq j$;

- Each local controller is well defined such that $C_{i}$ is stabilizing $M_{i}, i=1,2, \cdots, N$, and the output of the resulting closed-loop system $\left\{C_{i}, M_{i}\right\}$, say $y_{i}(k)$, is tracking the reference signal $y_{r}(k)$ in the sense that $\lim _{k \rightarrow \infty} \frac{1}{k} \sum_{r=1}^{k}\left[y_{i}(r)-y_{r}(r)\right]^{2}=R^{\prime}, R \leq R^{\prime}<\infty$.

then it is stable.

Proof. First, the weighting algorithm is convergent. So,

$$
p_{j}(k) \rightarrow 1 ; p_{i}(k) \rightarrow 0, i=1, \ldots, N, i \neq j ; p_{\theta}(k) \rightarrow 0
$$

where $p_{j}(k), p_{\theta}(k)$ index weights of the true model and the adaptive identification model.

Then, the VES for WMMAC in this case is similar to the one in Ref. 4 . The influence of the adaptive model on the system will be included in $\Delta u(k)$. And,

$$
e_{i}(k)=y(k)-y_{i}(k)=y(k)-\varphi^{\mathrm{T}}(k-d) \theta_{i}
$$

where $\varphi(k-d)=[-y(k-1), \cdots, u(k-d), \cdots, \hat{\omega}(k-1), \cdots]$.

Follow the proof process in Ref. 4, VES theory can be used to verify the stability.

Theorem 2. If a WMMAC system satisfies:

- The true model of plant isn't included in the fixed models of the model set $\mathrm{M}$;

- Model $M_{\theta}$ generates with probability one the minimum output error in the sense that

$$
\left\{\begin{array}{c}
\sum_{r=1}^{k} e_{\theta}^{2}(r)<\sum_{r=1}^{k} e_{i}^{2}(r), \forall k \geq d+1, i=1, \ldots, N \\
\frac{1}{k} \sum_{r=1}^{k} e_{\theta}^{2}(r) \rightarrow R_{\theta} ; \frac{1}{k} \sum_{r=1}^{k} e_{i}^{2}(r) \rightarrow R_{i}
\end{array}\right.
$$

where $R_{\theta}$ is a constant, $R_{i}$ may be constant or infinity, $R_{\theta}<R_{i}$;

- The adaptive controller $C_{\theta}$ is stabilizing $M_{\theta}$, and the output of the resulting closed-loop system $\left\{C_{\theta}, M_{\theta}\right\}$, say $y_{\theta}(k)$, is tracking the reference signal $y_{r}(k)$ in the sense that

$$
\lim _{k \rightarrow \infty} \frac{1}{k} \sum_{r=1}^{k}\left[y_{\theta}(r)-y_{r}(r)\right]^{2}=R^{\prime}, R \leq R^{\prime}<\infty .
$$

then it is stable.
Proof. First, the weighting algorithm is convergent. So,

$$
p_{\theta}(k) \rightarrow 1 ; p_{i}(k) \rightarrow 0, i=1, \ldots, N
$$

As the VES for this WMMAC is similar to the one in Ref. 8. The influence of the parameter identification process on the system will be included in $e_{i}(k)$.

Follow the proof process of Ref. 8, the VES for WMMAC can be decomposed into three subsystems. Then, VES theory can be used to verify the stability.

\subsection{WMMAC for parameter jump plant}

To consider the parameter jump plant, we assume that the system parameters are piecewise constant function of time, and time interval of parameter jump is long enough.

According to the assumptions, we know that each stage is a WMMAC for LTI plant. The overall system is slow switching between all the stages. Therefore, switching system theory ${ }^{9}$ can be utilized to verify the stability of the WMMAC system.

\section{Si mulation Studies}

In this section, the results of computer simulations will be presented. Consider an uncertain discrete-time plant

$$
\begin{gathered}
\left(1+a_{1} z^{-1}+a_{2} z^{-2}\right) y(k)=z^{-1}\left(b_{0}+b_{1} z^{-1}\right) u(k) \\
+\left(1+c_{1} z^{-1}\right) \omega(k)
\end{gathered}
$$

where $\omega(k)$ is obtained by $\operatorname{sqrt}(0.01) * \operatorname{randn}(1)$ in MATLAB,$c_{1}=0.5$. This discrete-time model can be obtained by the simple continuous-time in (18) with sample time $t_{s}=0.5 \mathrm{~s}$ and the zero order hold.

$$
G(s)=\frac{h}{s^{2}-3 s+2}
$$

For simplicity, four fixed local models are used as the uncertainty of $h$, that is, $h_{1}=0.7, h_{2}=0.8$, $h_{3}=1.2, h_{4}=1.3$. The four corresponded controllers are designed by pole placement control.

The expected closed-loop characteristic polynomial is corresponds to the characteristic polynomial of the following continuous-time second-order system

$$
\frac{\omega_{n}^{2}}{s^{2}+2 \xi \omega_{n} s+\omega_{n}^{2}}
$$

where $\xi$ is $0.7, \omega_{n}$ is 1 . 
Then we can know the four fixed controller easily. At the same time, the adaptive identification model is designed by indirect pole assignment self-tuning control. The recursive extended least square is used to estimated parameter. The initial value is $10^{6} *$ eye $(n a+n b+1)$. The calculation in detail is shown in formula (6) and (7).

The true model of plant is not included in the four fixed model, which $h$ is 1 . The initial value of adaptive model $\theta$ will affect the system performance.

The initial value $\theta=\left(\theta_{1}+\theta_{2}+\theta_{3}+\theta_{4}\right) / 4$.The simulation results are shown in Fig. 2 and Fig.3.

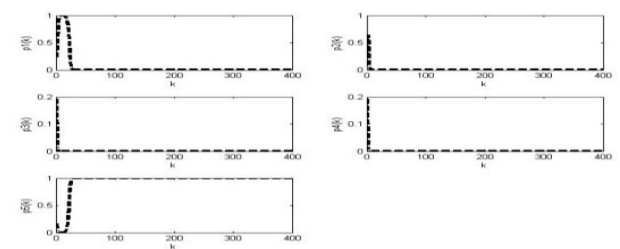

Fig. 2. Controller weight signals of case 1
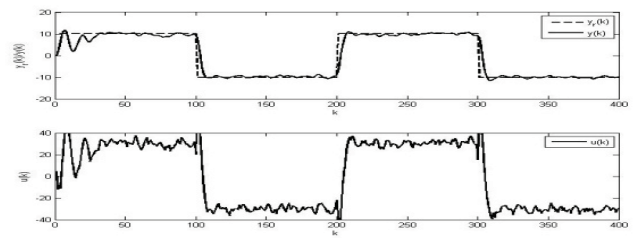

Fig. 3. Output, reference and control signals of case 1

The initial value is the parameter value of the fixed model closest to the real model. The simulation results are shown in Fig.4 and Fig.5.

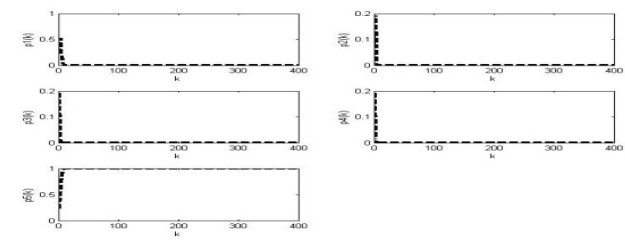

Fig. 4. Controller weight signals of case 2

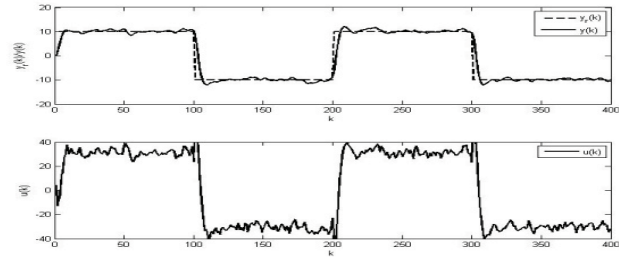

Fig. 5. Output, reference and control signals of case 2
In the two cases, the controller 5 designed according to the adaptive identification model all are chosen rapidly. However, the latter one works better.

\section{Conclusions}

In this paper, a finite number of fixed and one adaptive identification models are designed for a category of systems with colored noise. This method can effectively deal with issue when the real model isn't in the model set. And, the stability of WMMAC in the case is proven. At the same time, an adaptive model with real-time initial value, the parameter value of the fixed model closest to the real model, can improve the system performance.

\section{Acknowledgements}

This work was supported by National Natural Science Foundation of China (61520106010) and National Key Technologies R\&D Program (2013BAB02B07).

\section{References}

1. K. S. Narendra, X. Cheng. Adaptive control of discretetime systems using multiple models. IEEE Transactions on Automatic Control, 2000, 45(9): 1669-1686.

2. Moose $\mathrm{R}$ L, Van Landingham $\mathrm{H} F$, McCabe $\mathrm{D} H$ Modeling and estimation for tracking maneuvering targets. IEEE Transactions on Aerospace and Electronic Systems, 1979, AES-27: 448-456.

3. J. Chen, W. Chen, J. Sun. A survey of multiple model adaptive control. J. Sys. Sci. \& Math. Scis, 2014, 34(12):1421-1437.

4. W. Zhang. Stable weighted multiple model adaptive control: discrete-time stochastic plant. International Journal of Adaptive Control and Signal Processing, 2013, 27(7): 562-581.

5. F. Li. Robust multiple model adaptive control base on dynamic optimization model bank. Master Thesis, South China University of Technology, 2004.

6. W. Zhang. Weighted multiple model adaptive control of discrete-time stochastic system with uncertain parameters. Acta Automatica Sinica, 2015, 41(3): 541550.

7. Z. Pang, H. Cui. System identification and adaptive control MATLAB simulation. Beihang University Press, Beijing, China, 2009, pp:175-189.

8. W. Zhang. On the stability and convergence of selftuning control - virtual equivalent system approach. International Journal of Control, 2010, 83(5): 879-896.

9. Shorten R, Wirth F, Mason O, Wulff K, King C. Stability Criteria for Switched and Hybrid Systems. SIAM Review, 2007, 49(4): 545-592. 